\title{
Quantitative exposure assessment for confinement of maize biogenic systems
}

\author{
Jeffrey D. WOLT*, Yuh Yuan SHYY, Paul J. CHRISTENSEN, Karin S. DORMAN, Manjit MISRA \\ Biosafety Institute for Genetically Modified Agricultural Products, Iowa State University, Ames, IA 50011, USA
}

\begin{abstract}
The development of transgenic crops as production platforms for biogenic agents will largely depend on the success of efforts to confine the genes and their expressed proteins in field environments. We have used quantitative exposure assessment to evaluate how management practices affect materials escape due to outcrossing by pollen flow or grain loss during harvest operations. Specifically, we study the use of maize to produce biogenic agents within field-confined systems. Decision trees representing simplified schemes of fully conforming (designed to comply with current regulatory standards for field confined trials), partially conforming, and non-conforming management practices were developed. Exemplifying assumptions and published data for conformance and material fate probabilities were used in Monte Carlo simulations to forecast materials escape by pollen outcrossing and harvest operations from a 1 ha source field. Deterministic analyses showed fully conforming confinement management restricted materials loss to low levels (for this example, outcrossing produced $<1$ in $10^{6}$ kernels in receptor fields). The corresponding high-end (90th percentile) probabilistic result was 16- and 4333-fold higher (relative to deterministic outcrossing =1) for outcrossing and harvest loss, respectively. For partially conforming practice, high-end outcrossing ranged from $100-$ to $>15000$-fold over the base result in receptor fields, and harvest loss was $>10000$-fold over the base result. For non-conforming practice, high-end outcrossing produced $>15000$-fold greater kernels in receptor fields and high-end harvest loss was at least 19000-fold greater. Deterministic estimates of off-field loss by machine transfer are as much as 30000 -fold higher for non-conforming operations relative to the base case of pollen outcrossing. Better knowledge of failure frequencies for confinement management practices, improved physical models of materials flows, refined analysis of confinement loss probabilities using quantitative tools, and decision analysis to improve and audit management system performance are all needed to extend understanding of confinement integrity beyond the exemplifying case used here.
\end{abstract}

Keywords: transgenic / containment / plant manufactured pharmaceutical

\section{INTRODUCTION}

Since their relatively recent inception, the cultivation of crops expressing transgenic traits has become widespread. In 2003, more than 67.7 million hectares were planted worldwide with crops expressing transgenic traits (James, 2003). Despite their rapid adoption by producers, some public and regulatory questions remain regarding the long term impacts of these products (NRC, 2002). In order to manage uncertainties surrounding these technological innovations, and in response to earlier breakdowns in the management of transgenic trait deployment and distribution (Bucchini and Goldman, 2002), considerable care has been taken to ensure confinement of products lacking full regulatory approval. Despite these efforts, incomplete compliance with regulatory conditions for confinement of transgenic crop field experiments can occur (USDA, 2004; USEPA, 2002). Incomplete compliance is of special concern when food plants are used as biogenic platforms for pharmaceutical and industrial protein production, since these products are not intended to occur in foods or feeds. The concern intensifies when unintended gene flow results in gene replication within seed supplies used in food/feed production (Mellon and Rissler, 2004).

Within the United States, research into plant-based expression and field production of biopharmaceuticals

\footnotetext{
* Corresponding author: jdwolt@iastate.edu
} 
and industrial enzymes in food crops is moving at a rapid pace, but is hindered by uncertainties surrounding the ability to adequately confine biotechnology agents in field environments. The use of transgenic crop plants as in-field platforms for commercial production of biotechnology agents will require the establishment of highly reliable and verifiable means of confinement (FDA, 2003). In recognition of the need for increased vigilance in the confinement of experimental materials, USDA-APHIS (the responsible authority within the United States) has modified permitting conditions for field testing of plants engineered to produce either pharmaceutical or industrial compounds and has increased regulatory oversight to ensure compliance (USDA, 2003a; 2003b). Even so, questions and challenges remain regarding the adequacy of the confinement schemes that are being proposed (Ellstrand, 2003).

As described by Ellstrand (2003), no technology is risk free; therefore, the balance of societal risks and benefits must be considered when determining the appropriate safeguards and regulatory scrutiny needed to manage the technology. Risk assessment affords a formalized process for scientific evaluation of the risks posed by field confined plant biogenic pharmaceutical production (Peterson and Arntzen, 2004). Formalized risk assessment has broad applicability to innovations of biotechnology (Wolt and Peterson, 2000). In particular, quantitative risk assessment methods that describe the probability for harm under environmentally realistic exposures have been usefully applied to problems of modern biotechnology (Sears et al., 2001; Wolt et al., 2003). Well-recognized techniques for quantitative risk assessment, especially quantitative exposure assessment (see for example Cullen and Frey, 1999; Vose, 2000), allow scientifically sound evaluation of the confinement conditions necessary to meet risk management objectives for field-confined plant biogenic systems at all stages of development, from experimental trials through commercial production.

The objective of this study was to apply quantitative exposure assessment (QEA) to assess field confinement practices for maize as a biogenic production system using the available published data and exemplifying assumptions in the absence of data. Specifically, we report a preliminary assessment of the levels of outcrossing and harvest loss during commercial-scale field production when applying current recommended confinement practices for field-confined trials (USDA, 2003a; 2003b). For this study, we focus on three questions, summarized to what quantity of maize kernels expressing the biogenic trait:

1. escapes to non-source fields due to loss of pollen confinement;
2. remains in the source field seed reservoir after harvest, or

3. is left in the harvesting equipment?

\section{METHODS}

Quantitative exposure assessment constitutes an objective approach to evaluating the integrity of confinement strategies. QEA is a formalized process consistent with established framework approaches for risk management and policy decision-making (NRC, 1983; USEPA, 1992). The process entails an explicit statement of the problem; an appropriate conceptual model; an appropriate modeling approach; information to populate the model; and distributions to capture model input variance (heterogeneity) and uncertainty (incomplete knowledge). Results of the assessment include characterization of exposure probabilities, uncertainty about these probabilities, and the factors that significantly contribute to both (Cullen and Frey, 1999).

\section{Problem formulation}

A first step in ensuring that confinement conditions are met and that compliance oversight is appropriate is the unambiguous documentation of the requisite operational events involved in every phase of production of the biotechnology agent. The Biosafety Institute for Genetically Modified Agricultural Products (BIGMAP, www.bigmap.iastate.edu), of which we are a part, has used expert opinion to develop systematic flow diagrams of the major operations and underlying events necessary for plant biogenic pharmaceutical production in maize (Christensen et al., 2005). Analysis of the flow diagrams identifies possible sources of material outflows.

\section{Conceptual model}

The level of exposure and the severity of the hazard define the risk associated with the deployment of a transgenic crop. Confinement practices alter the process flows to limit exposure. This conceptual scheme is shown in Figure 1, where confinement practices are determined by the agronomic system, the environment in which it is deployed, and an a priori assumption regarding the hazard (or perceived hazard) for the transgenic trait.

To define the confinement practices, we consider the current regulatory guidance for field-testing of plants intended to produce pharmaceutical and industrial compounds (USDA, 2003a; 2003b). This guidance is uniformly applied to any pharmaceutical or industrial 


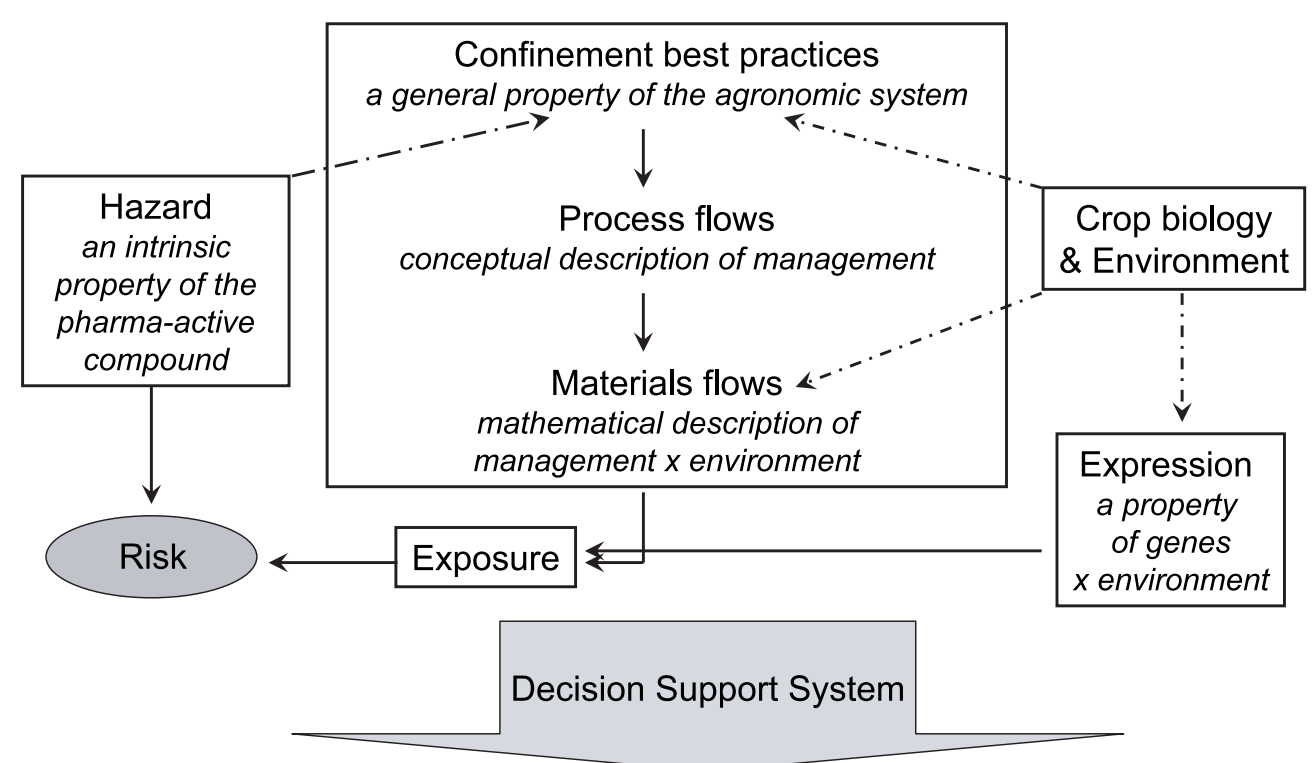

\section{Risk Management}

Figure 1. A conceptual model for the analysis of confinement practices as a component of exposure and risk for field-confined maize production systems. Solid lines represent direct flows of information and materials leading to estimates of risk. Dashed lines represent flows of knowledge that influence both product and confinement system design; thus, they indirectly influence estimates of risk.

compound under field confinement, and implicitly assumes high hazard of the biogenic agent, a cautionary approach taken in the face of little specific knowledge about the actual hazard. For the purposes of this exposure assessment we accept uncritically this approach, and adapt existing practices for foundation maize seed production (Christensen et al., 2005) to comply with the USDA guidelines. Our analysis considers the hierarchical processes necessary to limit outcrossing and harvest loss during field production of a plant biogenic agent expressed in maize (Tab. 1).

\section{Modeling approach, sources of information, and distributional assumptions}

We use a decision tree approach to translate process descriptions into logical sequences of activities determining the flow of products, by-products, and genetic information through the system (Figs. 2 and 3). The possible confinement activities pertaining to pollen flow and harvest management are listed in Table 2 along with the parameters and assumptions used to model them. Input distributions describing data variance and uncertainty are also indicated along with our definitions of partially conforming and nonconforming confinement activities. Input data were gleaned from published information, and do not represent an exhaustive review of the literature, but instead are intended to be representative of typical maize production in the Midwestern USA. In those instances where we use exemplifying assumptions, we have selected typifying values for the input that are consistent with current practices for maize seed production. The current screening level assessment is intended to demonstrate the decision tree approach within a quantitative assessment framework and was developed using TreeAge Pro software for model construction and deterministic analyses (release 5.1; TreeAge Software, Inc., Williamstown, MA) and Crystal Ball Professional analysis software (version 5.5, Decisioneering, Inc., Denver, $\mathrm{CO})$ for distributional analyses. Our example is for the simple consideration of a 1 ha confined source field and a 1 ha receptor field. Details of the model and parameter values are considered separately for outcrossing and harvest loss below. 
Table 1. Process activities for field-confined maize production used as the basis for quantitative exposure assessment of maize kernels from a confined field (after Christensen et al., 2005).

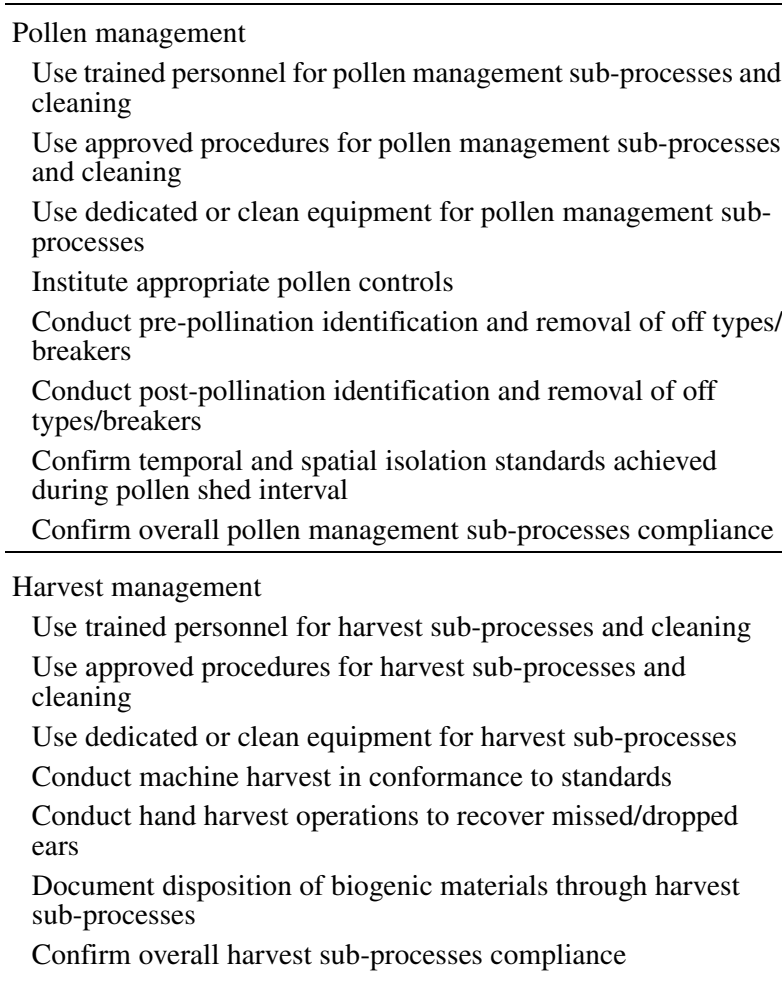

\section{Pollen management}

Pollen flow transmits genetic information from biogenic production fields to receptor fields within the vicinity. We evaluate unintended pollen flow by estimating the probability that a kernel in the receptor field is biogenic.

The data of Jones and Newell (1946) and Jones and Brooks (1950) represent widely cited outcrossing measurements for maize conducted under worst case conditions of fully receptive (flowering) plants and unimpeded wind flow. Following the approach of Aylor et al. (2003), we modeled these data using a Cauchy-like function to represent viable outcross fraction $(O C)$ as a function of distance

$$
O C(D)=\frac{b_{0}}{1+\left(\frac{D}{b_{1}}\right)^{b_{2}}},
$$

where $D$ is distance in meters, $b_{0}=0.288 \pm 0.022, b_{1}=$ $17.5 \pm 4.13$, and $b_{2}=1.6 \pm 0.46$. We assume the plant is homozygous with respect to the trait of interest in keeping with current plant manufactured pharmaceutical production practice (personal communication Kan Wang, Iowa State University Plant Transformation Facility). With this model, the predicted outcrossing is $0.021 \%$ at the $1610 \mathrm{~m}$ (1 US mile) offset prescribed by the USDA guidelines for crops expressing biopharmaceutical traits (USDA, 2003a).

Fully conforming conditions include both temporal and spatial offsets and require no receptive maize to occur within 1610 m of the source field within \pm 28 days of pollen shed at the source (USDA, 2003a). We conservatively estimate $O C$ for fully complying fields by assuming synchronous flowering in source and receptor fields, and assuming that all plants in the receptor field stand $1610 \mathrm{~m}$ distant from the source field. Indeed the predicted $O C$ is conservative compared to other published data for maize pollen dispersal (Fig. 4; see, for instance, Feil and Schmid, 2002). We define non-conforming management as the presence of a receptive maize field at a sub-optimal distance $(<1610 \mathrm{~m})$, again with complete temporal overlap. Without further data on compliance failure rates, non-conforming spatial offsets are unknown. To model this uncertainty, we randomly sample a uniform distribution of distances from 1 to $1610 \mathrm{~m}$ offset from the source field for estimation of $O C$ (Tab. 2).

The extent of outcrossing is further limited by use of male sterile plants in conjunction with detasseling operations to reduce source pollen as shown in the decision tree for pollen management operations (Fig. 2). We assume the outcrossing fraction is directly proportional to the amount of source pollen, so a reduction in source pollen reduces the outcrossing proportionally. In the present analysis, we model uncertainty in the effectiveness of the male sterility system using a triangular distribution (Tab. 2) where the modal effectiveness is 0.99 (range 0.80 to 0.999 ) under conforming managements and 0.50 (range 0 to 0.999 ) when non-conforming. Uncertainty in the efficiency of conforming and non-conforming detasseling practices are similarly represented by triangular distributions with modal effectiveness 0.95 (range 0.90 to 0.999 ) and 0.50 (range 0 to 0.999 ), respectively (Tab. 2). Empirical data to populate the model were limited; therefore, input assumptions reflect both limited knowledge on the magnitude of input variance and uncertainty about the consequences of conformance failure.

\section{Harvest management}

Field harvest operations commence from the point of preharvest verification and removal of off types (Christensen et al., 2005). Harvest operations contribute to maize kernels left within the field (a concern relative to volunteers 


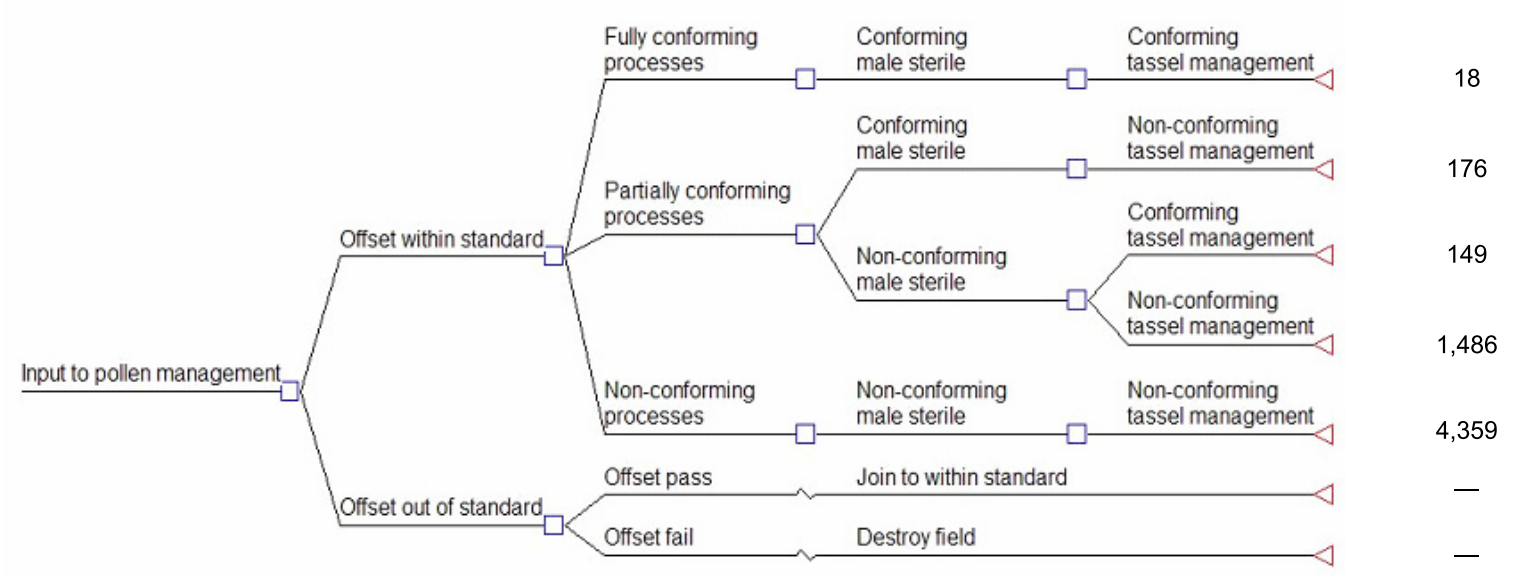

Figure 2. Decision tree for pollen flow from confined field production of maize and 50th percentile outcomes for an exemplifying case of confinement management for a 1 ha source field with pollen outcrossing to a 1 ha receptor field. Input to the pollen management process begins with an initial determination of the appropriate offset distance (i.e., "offset within standard") between the source and receptor field. For an "out of standard" field, if the offset is corrected ("offset pass"), it can return to within standard; otherwise ("offset fail") the source field is destroyed. For a source field within standard, subsequent conformance to temporal offset conditions is considered.

${ }^{a}$ Outcrosses arising from pollen flow to a 1 ha receptor field producing 20128000 seeds.

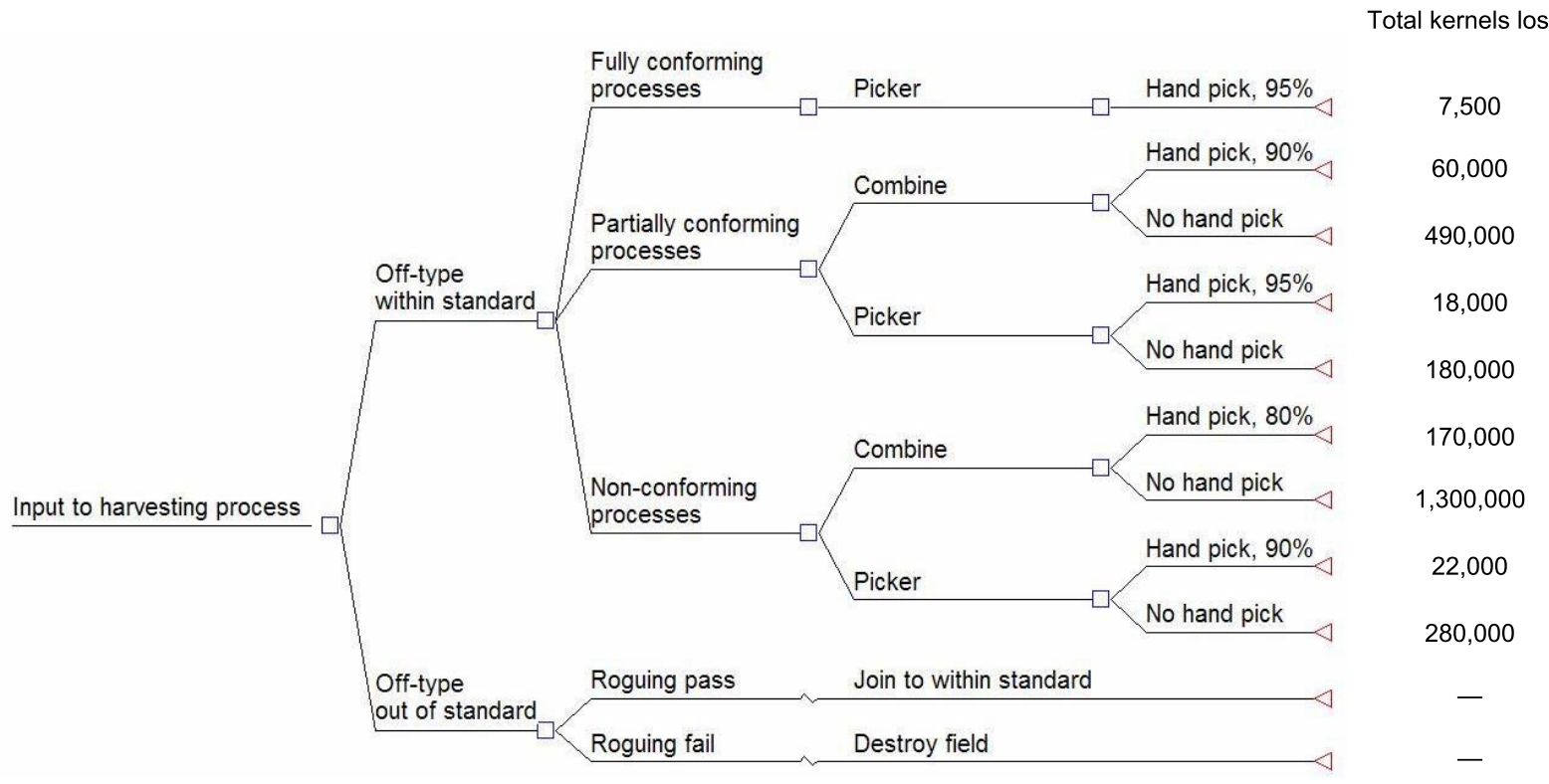

Figure 3. Decision tree for total harvest loss of grain from confined field production of corn and 50th percentile outcomes for an exemplifying case of confinement management for a 1 ha field. Input to the harvest management process begins with a determination of whether the proportions of off-type plants within the source field conform to the biogenic production standard ("off-type within standard"). If the off-type is out of standard and can be corrected by rouging of the field ("rouging pass"), it can return to within standard; otherwise ("rouging fail") the source field is destroyed.

${ }^{a}$ Lost kernels from a 1 ha source field producing 20128000 seeds. 
J.D. Wolt et al.

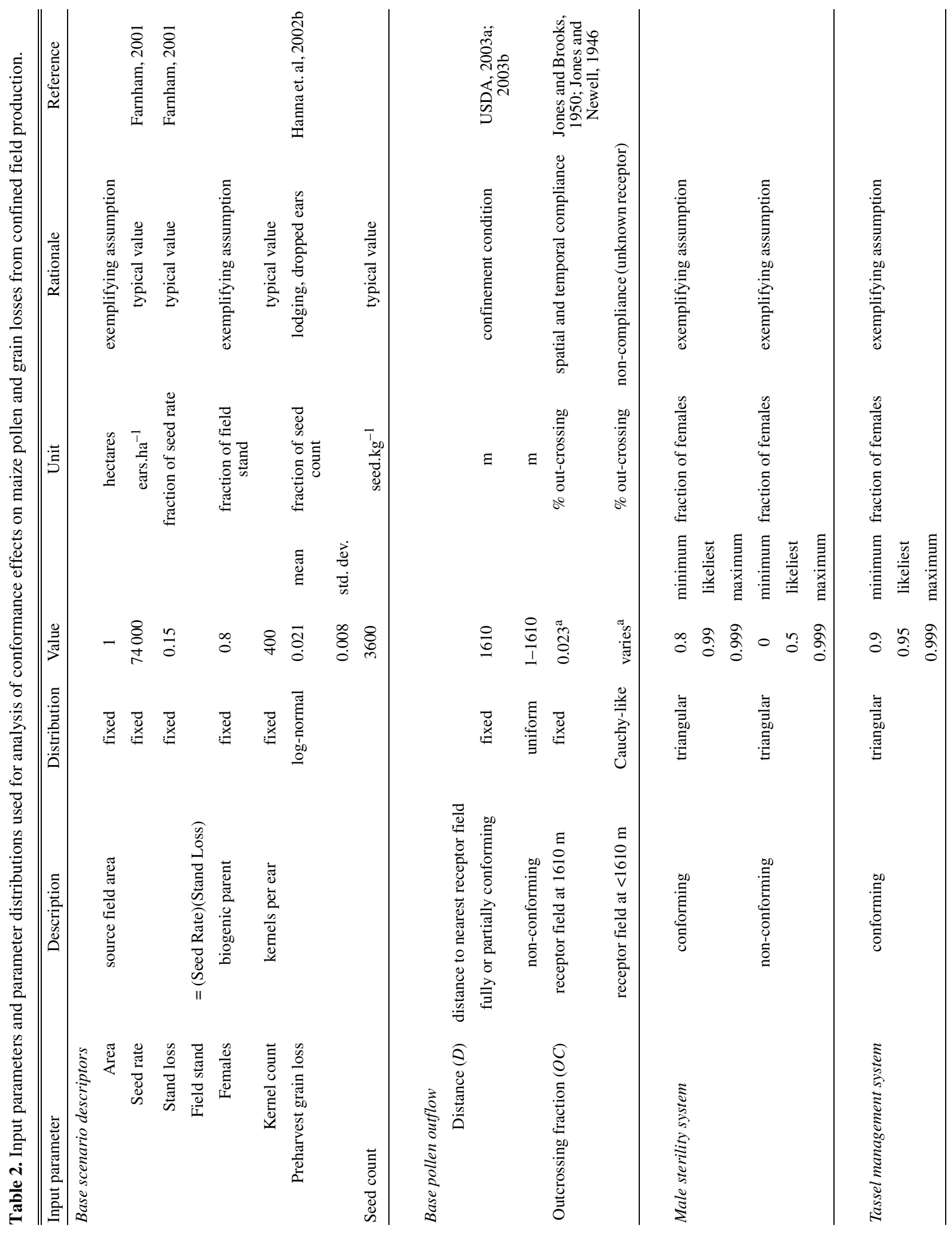


Assessing maize confinement

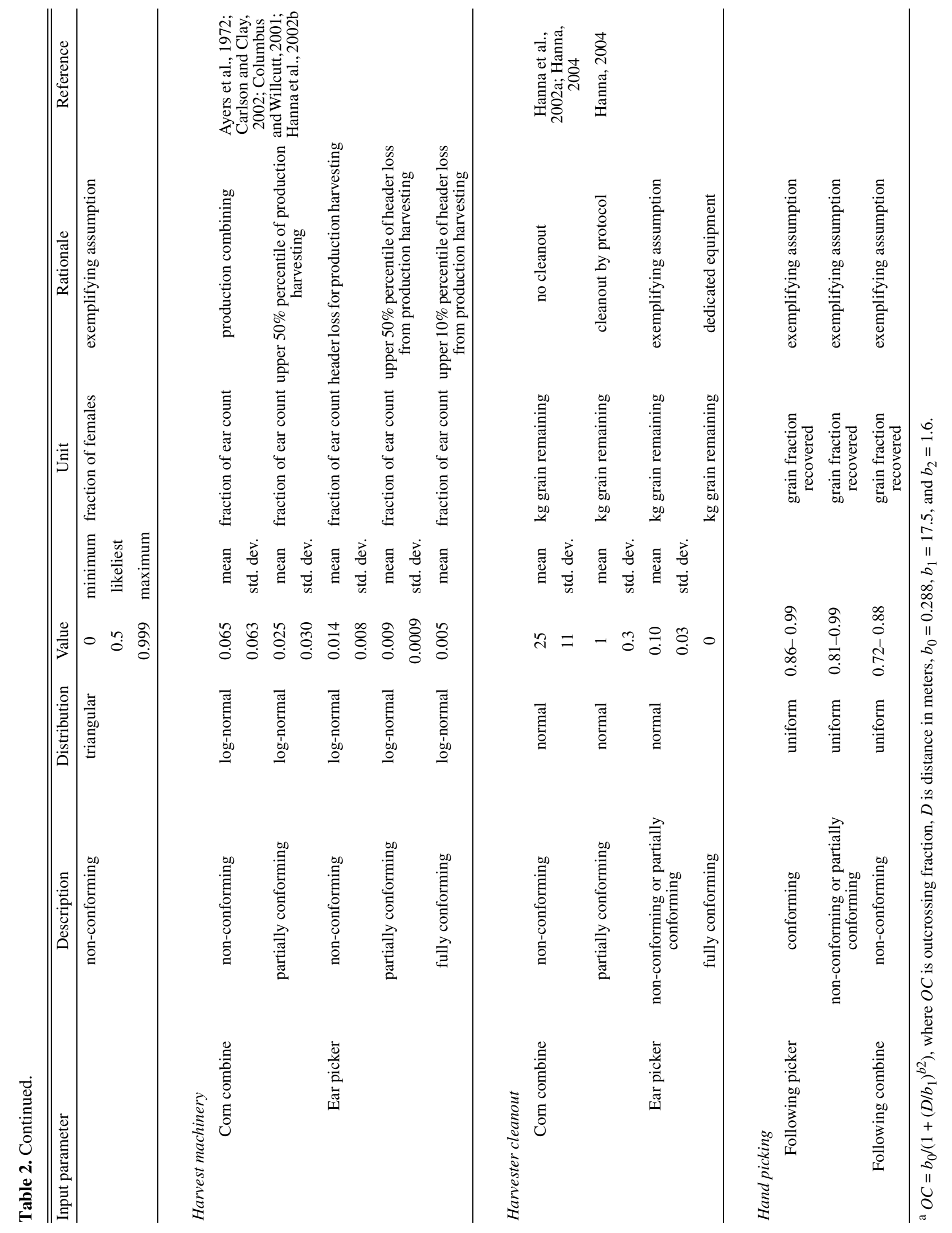


in subsequent years, fate of kernels in soil, and removal of kernels by mammals and birds) and to unintended transmission of genetic information through harvest equipment. If pre-harvest standards are met, then harvest can be conducted and is assumed to follow the decision tree shown in Figure 3. Studies describing maize loss from machine harvest operations were used to develop distributions for harvest loss (Ayers et al., 1972, as cited in UACES, 2003; Carlson and Clay, 2002: Columbus and Willcutt, 2001; Hanna et al., 2002b). Composite data representing measurements of total visible loss for typical production equipment were used as follows: preharvest dropped ears represented losses due to lodging (93 total measurements); machine ear loss (loss at the header) was considered as representative of picker losses (94 measurements); and total visible machine loss (the sum of machine ear, stalk roll shelling, cylinder, and separating losses) represented combine losses (184 measurements). Log-normal distributions were determined to adequately represent all of these forms of harvest loss. We develop one distribution to describe preharvest loss independent of confinement procedure. Another represents non-conforming machine harvest loss and is based on combine data. The lower 50th percentile of either picker or combine losses were used to develop distributions for partially conforming machine harvest. Fully conforming machine harvest loss was determined using data from the lower decile of picker loss (Tab. 2). We view these estimates of machine harvest efficiency as conservative, since they are developed from data for harvest operations using production equipment where no special efforts were made to calibrate the equipment to harvest conditions.

Appropriate management practices for crop confinement include hand picking following machine harvest to recover ears lost in the field during mechanical harvest (Christensen et al., 2005). There is little known about the efficiency of hand picking procedures, so we define distributions to account for this uncertainty. We assume efficiency of hand picking after machine harvest with a huskon ear picker follows a uniform distribution (for fully conforming practices: midpoint 0.925 , range $0.86-0.99$; for partially conforming or non-conforming practices: midpoint 0.90 , range $0.81-0.99$ ). Similarly, for partially conforming or non-conforming hand picking following machine harvest with a combine, a uniform distribution with midpoint 0.80 and range $0.72-0.88$ is used.

\section{Machine cleaning}

Finally, crop confinement management practices include machine cleaning to prevent outflow of biogenic plant residue via mechanical transport. Maize combines subject to typical use contain $45 \pm 11 \mathrm{~kg}$ of plant residues after harvest operations; approximately $50 \%$ of this represents grain (25 $\pm 6 \mathrm{~kg}$ grain) (Hanna, 2004; Hanna et al, 2002a). These data are considered typical of non-conforming harvester clean-out procedures in our analyses. Partially conforming procedures should subject the combines to systematic cleaning, where grain carryover is reduced to about $1.0 \pm 0.3 \mathrm{~kg}$ (Hanna, 2004). We estimate for both partially and non-conforming harvester clean-out procedures that husk-on pickers contribute only $0.1 \mathrm{~kg}$ grain due to the greater simplicity of the clean-out procedure for pickers versus combines.

\section{Probabilistic assessment of confinement practice}

Distributional analysis was conducted to evaluate possible outcomes. The outcome distributions represent the effect of both model input uncertainty and process variability. Ten thousand Monte Carlo iterations, involving random sampling from the input distributions (Tab. 2), were conducted to forecast outcrossing and harvest loss distributions for each of our fully conforming, partially conforming, and non-conforming cases of confinement management.

\section{RESULTS}

\section{Pollen management}

Pollen confinement was measured as the probability that a kernel in the receptor field arises from outcrossing with fugitive pollen from the source field. In the absence of parameter uncertainty, our model sets this probability as

$$
\left(1-s_{c}\right)\left(1-t_{c}\right) O C(D)
$$

where $s_{c}$ is the male sterility efficiency, $t c$ is the detasseling efficiency, and $O C(D)$ is the previously described outcross fraction as a function of receptor offset distance $(D)$. Subscript $c$ indicates the parameter value depends on the confinement condition selected.

When fully conforming conditions (spatial offsets with redundant physical and biological source pollen control) are followed, point estimates for $D, s_{c}$, and $t_{c}$ are $1610 \mathrm{~m}, 0.99$, and 0.95 , representing the prescribed offset distance and the most likely values for sterility and detasseling efficiency. On the basis of these inputs, the probability of an outcrossed kernel is $<1$ biogenic kernel 
Assessing maize confinement

Table 3. Relative predicted materials $\operatorname{loss}^{\mathrm{a}}$ in relation to degree of conformity to production management practices.

\begin{tabular}{|c|c|c|c|c|}
\hline & & \multirow{2}{*}{$\begin{array}{l}\text { Deterministic } \\
\text { result }\end{array}$} & \multicolumn{2}{|c|}{ Distributional result } \\
\hline & & & 50th percentile & 90th percentile \\
\hline \multicolumn{5}{|c|}{ Outcrossing (to nearest receptive field) } \\
\hline \multirow{2}{*}{$\begin{array}{l}\text { Fully conforming } \\
\text { Partially conforming }\end{array}$} & & $1^{\mathrm{b}}$ & 6 & 16 \\
\hline & Male sterility system & & 59 & 166 \\
\hline \multirow[b]{3}{*}{ Non-conforming } & Detasseling & & 50 & 100 \\
\hline & Male sterility system + Detasseling & & 500 & 1000 \\
\hline & & & 1467 & 15333 \\
\hline \multicolumn{5}{|c|}{ 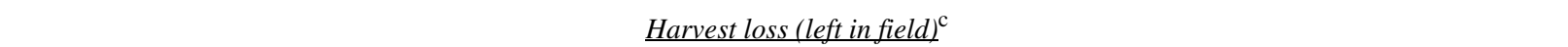 } \\
\hline $\begin{array}{l}\text { Fully conforming } \\
\text { Partially conforming }\end{array}$ & & $2500^{\mathrm{d}}$ & 2500 & 4333 \\
\hline \multirow{5}{*}{ Non-conforming } & Combine & & 20000 & 73333 \\
\hline & Ear picker & & 6000 & 10333 \\
\hline & & & & \\
\hline & Combine & & 60000 & 176667 \\
\hline & Ear picker & & 7333 & 19000 \\
\hline \multicolumn{5}{|c|}{ Harvest loss (harvest mixing) } \\
\hline \multirow{3}{*}{$\begin{array}{l}\text { Fully conforming } \\
\text { Partially conforming }\end{array}$} & & nil & nil & nil \\
\hline & & & & \\
\hline & Ear picker & 120 & & \\
\hline Non-conforming & & 30000 & & \\
\hline
\end{tabular}

per million. Relative comparisons to this point estimate (our base case) with the results of distributional analyses for various degrees of management conformance are presented in Table 3. Distributional analysis for the conforming case (when uncertainty about $s_{c}$ and $t_{c}$ are described by triangular distributions, Tab. 2) provided a somewhat higher result (6- and 16-fold higher for the 50th and 90th percentile outcomes, respectively), due to the skew in the distributions describing $s_{c}$ and $t_{c}$. In comparison, when spatial offsets are achieved but only one fullyconforming pollen management practice is employed, distributional analysis indicated biogenic occurrence in a receptor field rises slightly to (50-fold for the 50th percentile result when conforming male sterile systems are used with non-conforming tassel management, and 59fold when conforming tassel management is used with non-conforming male sterile systems). When spatial offset is uncertain and pollen management operations are non-conforming, the 50th percentile biogenic presence in a receptor field is 1467 -fold higher than the base case.

\section{Harvest management}

The probability that a kernel is lost in the field as a result of harvest management operations is

$$
m_{c}\left(1-h_{c}\right)
$$

where $m_{c}$ is the efficiency of mechanical picking and $h_{c}$ is the fraction of lost grain recovered with hand-picking. With point estimates $m_{c}=0.005$ and $h_{c}=0.925$, the deterministic estimate of harvest loss under the fully conforming conditions is 2500 -fold the deterministic result for outcrossing. Distributional analysis for harvest loss with fully conforming practices forecasts 50th percentile and high-end harvest losses 2500-fold and 4333-fold higher than that for outcrossing (Tab. 3). In comparison, various scenarios for partially conforming or non-conforming management show high-end total harvest losses as much as $>10000$-fold higher for the unusual case of non-conforming operations using an 
improperly managed combine. These estimates represent partially conforming or non-conforming practices that include a hand pick operation; without a hand pick operation harvest loss increases by about an order of magnitude (Fig. 3).

Grain loss from confined field harvest operations will meet two fates. It will either remain in the source field, as described in the foregoing analysis or it will be carried from the field in harvest machinery. Kernels leaving the confined field in the harvester may be introduced to other fields where the equipment is used. A worst case would involve unintended introduction of maize from the confined field into seed harvested in another field by mixing seed with residual kernels in the harvester. Based on estimates of clean-out efficiencies (and assuming 3600 kernels per $\mathrm{kg}$ ), deterministic estimates of potential biogenic occurrence by machine transfer (relative to the base case of pollen outcrossing) range from 120-fold higher for a partially conforming picker, to 1200 -fold for a partially conforming combine harvester, and 30000fold for a non-conforming combine harvester. Under fully conforming practices the equipment would be dedicated, thus eliminating grain transfer by machine out of the confined production system.

\section{DISCUSSION}

In this analysis we have selected simple empirical models for pollen flow and biomass production to describe materials flows through the confinement system. The materials flow descriptions, parameter estimates, and quantitative descriptions of estimate uncertainties result in predictions of the probability of off-source or residual in-field transgenic kernels. Our estimates of off-source and in-field presence are intended to be conservative, since we have sought high-end empirical estimates of parameters for pollen flow, harvest efficiency, and plant residue containment. These descriptions are adequate as a conservative first attempt to describe the relative impact of conforming and non-conforming confinement management operations and to understand the impact of uncertainty. The methodology described is amenable to more exacting physical models for materials flows leading to site- and product-specific exposure estimates (see for instance pollen flow models such as Arritt et al., 2005; Colbach et al., 2001; Klein et al., 2003).

A principal concern for field trials of crops expressing biogenic traits is the possibility of gene escape followed by undetected replication within the seed production system for food crops. Gene flow from confined fields may result from pollen outcrossing to a receptor field or by transmission of viable kernels to seed lots through field operations (principally harvest operations). As shown in our calculations (Tab. 3), harvest loss in the field far exceeds outcrossing as a source of gene escape except when all pollen control measures are non-conforming. When pollen control measures are not documented and therefore do not conform, input uncertainty becomes overwhelming. In this instance, the center of the distribution still predicts fewer outcross kernels than harvest loss kernels, but the long tail of uncertainty extends beyond many harvest loss predictions. Lack of data has led us to approximate these parameter estimates, but the fact that pollen escape almost always produces less transgenic contamination than harvest loss suggests that control of field harvest operations needs special emphasis in terms of mitigating gene escape. In practice, however, it is possible to tightly control the amount of harvest loss material that leaves the field. Harvest cross-mixing can be minimized through procedures that include dedicated equipment and fields as well as physical inspection to verify compliance; whereas for pollen flow, physical evidence of off-source movement is mainly restricted to DNA analysis and is less likely to be observed.

We have not considered here other sources of unintended transmission of genetic information due to other crop management operations, such as sowing and tillage, and to the transport of harvested materials from fields to storage or process locations. We have described these processes elsewhere (Christensen et al., 2005), and they remain a consideration for our continuing evaluations of confinement integrity.

The relative concentrations of inadvertent biogenic material occurring outside of confinement depend on source and receptor field sizes. In our analysis we have fixed the source field size at 1 ha, which matches the data we used for relating outcrossing with source/receptor field separation. Thus, the effects of receptor field size, dimension, and orientation are not considered in this assessment. As shown by more complex models for particle dispersal from a source (see for instance USEPA, 2003), an increase in source field size will increase the potential for pollen outflow and subsequent unintended presence in a receptor field; whereas, an increase in the receptor field size will have a varied effect depending on size, depth, and orientation relative to the source field. Since we conservatively treat the whole field as sitting at a fixed distance from the source field, our outcrossing estimates are high. Still, we anticipate the variation in outcrossing resulting from reasonable combinations of source and receptor field sizes and positions will generally 


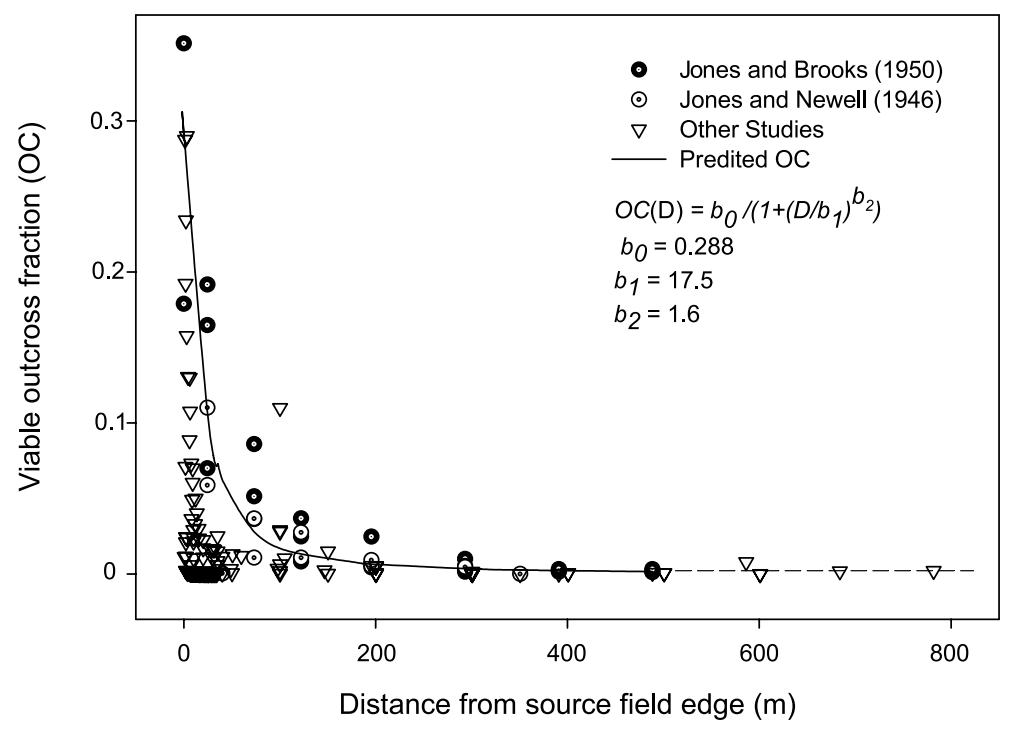

Figure 4. Empirical estimate for maize pollen viable outcrossing as a function of distance from a pollen source. The predicted outcross fraction was fit to the data of Jones and Newell (1946) and Jones and Brooks (1950), and is compared to other studies which report maize pollen flow and outcrossing (from multiple sources as summarized in Feil and Schmid, 2002). be less than the variation we observed between levels of conformance (Tab. 3). Harvest cross-mixing from biogenic seed will be insensitive to source field size, but the size of the seed lot it adulterates will influence relative concentration in the cross-mixed seed.

The empirically-derived function we generated for unrestricted pollen flow resulting in outcrossing as a function of receptor field distance was designed to conservatively overestimate outcrossing potential, especially since we assume a perfect temporal overlap in source and receptor fields. As suggested by the aerobiological framework of Aylor et al. (2003), we fit empirical data to a powerlaw function that reflects the "apparent long-extending 'tail' of the dispersal distribution." The modeled distribution (Fig. 4) predicts an outcrossing percentage of $0.58 \%$ at $198 \mathrm{~m}$, comparing favorably to the estimate $0.75 \pm 0.63 \%$ developed from diverse, although limited, data (from multiple sources as cited in Feil and Schmid, 2002). Yet, since data are limited for outcrossing to receptive maize at offset distances over $1610 \mathrm{~m}$, the utility of the power-law function and the data from which our relationship was developed remains uncertain when applied at conforming distances.

Even with spatial offsets in place, use of both biological (male sterile systems) and physical (tassel removal) methods are needed in our forecasts to minimize the potential for outcrossing due to off-source pollen flow. For our discrete example of fully conforming practice, the combined efficiency of a male sterile system and detasseling was $>99 \%$, yielding an outcrossing frequency consistent with expectations for high-quality foundation seed production (0.001; NRC, 2000), which served as the model for operations described here. The assumptions concerning pollen management efficiencies are consistent with our knowledge of current foundation seed production practices, but uncertain knowledge of these controls has lead others to conclude further data are needed on pollen management procedures and failure frequencies (especially with respect to bioconfinement; NRC, 2004).

Plant residues remaining in the field may pose a concern for non-target exposure within the source field, and some amount may also be inadvertently carried from the field. We are considering here an example of a biogenic material expressed within the maize kernel; but because of leakage effects, there remains the potential for some level of biogenic occurrence in the plant residue. These residues are managed in post-harvest operations and in the following growing season, and are principally of consequence with respect to potential ecological effects (such as to foraging animals and decomposition by soil organisms). Following the decision tree for harvest management (Fig. 3), the quantity of residues present following harvest varies amongst the scenarios. The total above ground biomass left in the field is comprised of leaves and stalks in all scenarios; it also includes some additional husk and cob material in those cases where harvest operations are either nonconforming or partially conforming. The decline in the field confined residues at the end of the following cropping cycle (one year later in this instance) could be determined under the assumption of a constant residue decline rate. Decline rates are uncertain but can be reasonably approximated as a first instance using a DT50 $=0.7 \mathrm{yr}$ as suggested by Jenkinson (1988). 
In our analysis, output variance is greater for partially and non-conforming than for fully conforming confinement practices. Increased output variance reflects both lack of knowledge and variance in the confinement procedures. Knowledge of the magnitude of output variance associated with confinement practices is important in order to control biogenic kernels below a pre-defined threshold, but even if knowledge were available, inherent heterogeneities would persist and contribute to output variance. For example, we lack knowledge of the likely spatial offsets in use when regulatory standards are not met, yet even with complete data, no single source/receptor field arrangement could describe all non-conforming spatial offset conditions. The goal of QEA is to identify conditions where either because of lack of scientific knowledge or imperfect confinement procedures, there is some non-negligible chance of producing more biogenic kernels than the threshold. In the first case, more scientific knowledge improves understanding of the adequacy of confinement procedures, whereas for the second case altered practices may be required to reduce inherit variance and assure confinement integrity. Improvements could include better quality control and quality assurance procedures or added redundancies in selected sets of tasks need to confine the biogenic products.

When even conservative estimates establish a particular procedure as satisfactory, or highly unlikely to produce fugitive biogenic kernels above the established threshold, then it becomes important to know the probability of failure to conform with this procedure, and given failure to conform, the distribution of the nonconforming inputs. In our analysis, we identified three types of protocols, (1) fully conforming, (2) partially conforming, and (3) non-conforming, and defined input distributions for each using data and expert opinion. We have made no attempt to quantify the relative probability of each protocol type and note that actual confinement practices will fall on a continuum. Lack of data on failure rates for the operations described prohibits us from evaluating process failure frequencies. Our analysis suggests that if our definitions of confinement practices are reasonable, the output variance between these three scenarios is far larger than the output variance within a given scenario (Tab. 3), leading to the conclusion that the threat of non-conformance may be far more important for outcrossing and harvest loss than the variability intrinsic in the processes themselves. In a sense, we expect greater variability in outcome between implementations of a containment protocol than between multiple years of the same implementation. Further analysis of these processes for transgenic maize should consider what kinds of nonconformance are likely and how non-conformance impacts input parameters.

This analysis identifies numerous uncertainties that need clarification before refined QEA is possible. We have used conservative estimates of biomass production and pollen dissemination. Tighter estimates require more exacting physical and biological models based on data collected from relevant environments. Such information is available, but refining the estimates downward has little importance when the degree to which confinement practices conform to standards is unknown.

A major limitation in our analysis is that source data is limited in many instances, which has led us to use a number of exemplifying assumptions. The input distributions we have selected for the current analysis (Tab. 2) reflect the richness of input data. Where data were largely lacking, uniform input distributions were used; where we had greater knowledge of inputs, triangular distributions were used; and where source data were sufficiently rich, we developed best fit distributions. As we have discussed, data limitations will need to be overcome before the present assessment can be refined and model robustness can be fully evaluated.

Improving the knowledge of residual uncertainties in field confined production of plant-expressed biogenic materials will benefit from increased understanding of process failure frequencies for conformance to confinement management standards, further analysis of confinement loss probabilities using quantitative tools, and use of decision analysis to understand how management systems may be improved and audited for performance. Non-conforming confinement practices are difficult to predict, but quantitative exposure assessment can help to identify practices and failure rates at particular stages of production that can substantially increase the ultimate exposure, and consequently risk. Additionally, concrete models can be used to customize management practices and regulatory standards for compliance. For example, it is possible to identify the required efficiency of male sterility and detasseling that could control outcrossing and allow for a smaller offset distance. Policy making should be improved with increased understanding of these uncertainties, as well as the understanding of the relative importance of non-conforming confinement management operations within strictly regulated field-confined biogenic systems. QEA represents an objective approach to evaluating the integrity of confinement strategies.

We have focused in this paper on the quantitative analysis of exposure arising from confined field production of maize. We have not addressed the 
consequences of losses from confinement, which would consider the hazard associated with the specific biogenic agent being produced by the maize. We have shown that confinement losses can be managed to very low thresholds of exposure, but the determination of likelihood of harm that may occur as a consequence of low level losses from confinement must be addressed within the context of product-specific risk assessments that account for the combined probability of exposure and hazard.

Received October 6, 2004; accepted February 28, 2005.

\section{REFERENCES}

Arritt RW, Clark CA, Reese JM, Westgate ME (2005) Lagrangian numerical simulations of maize pollen dispersion and comparison with observations. Intl. J. Biometeor. (in review)

Ayers GE, Babcock CE, Hull DO (1972) Corn combine field performance in Iowa. In Grain Damage Symposium, The Ohio State University, Columbus, Ohio, pp 1-17

Aylor DE, Schiltes NP, Shields EJ (2003) An aerobiological framework for assessing cross-pollination in maize. Agric. Forest Meteorol. 119: 111-129

Bucchini L, Goldman LR (2002) Starlink maize: A risk analysis. Environ. Health Persp. 110: 5-13

Carlson G, Clay D (2002) Estimating harvest loss. Accessed electronically 13 May 2004, http://plantsci.sdstate.edu/ precisionfarm/papers/harvest_loss\%202002.pdf

Christensen P, Manjit MS, Rai S, Shyy Y-Y, Wolt JD (2005) Confined Production Processes for Non-Food Corn. Biosafety Institute for Genetically Modified Agricultural Products, Iowa State University, Ames, Iowa

Colbach N, Clermont-Dauphin C, Meynard JM (2001) GENESYS: a model of the influence of cropping system on gene escape from herbicide tolerant rapeseed crops to rape volunteers - I. Temporal evolution of a population of rapeseed volunteers in a field. Agric. Ecosyst. Environ. 83: 235-253

Columbus E, Willcutt $\mathbf{H}$ (2001) Effect of field dry down and delayed harvest on corn yield and quality. MAFES Information bulletin 386. Accessed electronically, 13 May 2004, http://msucares.com/nmrec/reports/2001/corn/ production/effect.pdf

Cullen AC, Frey HC (1999) Probabilistic Techniques in Exposure Assessment: A handbook for dealing with Variability and Uncertainty in Models and Inputs. Plenum Press, New York

Ellstrand NC (2003) Going to "great lengths" to prevent the escape of genes that produce specialty chemicals. Plant Physiol. 132: $1-5$
Farnham D (2001) Corn planting guide. PM1885. Iowa State University Extension. Accessed electronically 5 July 2004, http://extension.iastate.edu/Publications/PM1885.pdf

Feil B, Schmid JE (2002) Dispersal of maize, wheat, and rye pollen. Shaker Verlag, Aachen, Germany

Food and Drug Administration [FDA] (2003) Guidance for Industry: Drugs, Biologics, and Medical Devices Derived from Bioengineered Plants for Use in Humans and Animals, Draft Guidance. FDA, Center for Biologics Evaluation and Research. Accessed electronically, 25 Feb 2004, http:// www.fda.gov/cber/gdlns/bioplant.htm

Hanna HM (2004) Combine clean-out research. 26th Annual Seed Technology Conference, Output Traits for Corn and Soybeans, 17 Feb 2004, Seed Science Center, Iowa State University

Hanna HM, Jarboe DH, Quick GR (2002a) Field equipment clean-out for identity-preserved grain production. Iowa Grain Quality Initiative, Iowa State University. Accessed electronically, 28 June 2004, http://www.extension.iastate.edu/Pages/grain/ publications/grainproduction.html

Hanna HM, Kohl KD, Haden DA (2002b) Machine losses from conventional versus narrow row corn harvest. Appl. Engineer. Agric. 18: 405-409

James C (2003) Preview: Global Status of Commercialized Transgenic Crops: 2003. ISAAA Briefs No. 30. International Service for the Acquisition of Agri-biotech Applications: Ithaca, NY

Jenkinson DS (1988) Soil organic matter dynamics, In Wild A. ed, Russell's Soil Conditions and Plant Growth, 11th edn, Longman, Essex, England, pp 564-607

Jones MD, Brooks JS (1950) effectiveness of distance and border rows in preventing outcrossing in maize. Oklahoma Agric. Exp. Sta. Tech. Bull. No. T-38. Stillwater, OK

Jones MD, Newell LC (1946) Pollen cycles and pollen dispersal in relation to grass improvement. Univ. Nebraska Agric. Exp. Sta. Res. Bull. No. 148. Lincoln, NE

Klein EK, Lavigne C, Foueillassar X, Gouyon PH, Larédo C (2003) Corn pollen dispersal: mechanistic models and field experiments. Ecological Monographs 73: 131-150

Mellon M, Rissler J (2004) Gone to Seed: Transgenic Contaminants in the Traditional Seed Supply. UCS Publications, Cambridge, MA

National Research Council [NRC] (1983) Risk Assessment in the Federal Government: Managing the Process. National Academy Press, Washington, DC

National Research Council [NRC] (2000) Genetically Modified Pest-Protected Plants: Science and Regulation. National Academy Press, Washington, DC

National Research Council [NRC] (2002) Environmental Effects of Transgenic Plants. National Academy Press, Washington, DC 
National Research Council [NRC] (2004) Biological Confinement of Genetically Modified Organisms. National Academy Press, Washington, DC

Peterson RKD, Arntzen CJ (2004) On risk and plant-based biopharmaceuticals. Trends Biotechnol. 22: 64-66

Sears MK, Hellmich RL, Stanley-Horn DE, Oberhauser KS, Pleasants JM, Mattila HR, Siegfried BD, Dively GP (2001) Impact of Bt maize pollen on monarch butterfly populations: A risk assessment. Proc. Natl. Acad. Sci. U.S.A. 98: $11937-11942$

United States Department of Agriculture [USDA] (2003a) Field testing of plants to produce pharmaceutical and industrial compounds. Federal Register 68 (46) 11337-11340

United States Department of Agriculture [USDA] (2003b) Introductions of plants genetically engineered to produce industrial compounds. Federal Register 68 (151) 4643446436

United States Department of Agriculture [USDA] (2004) What is the conformance history with APHIS' biotechnology regulations? USDAS, APHIS, Biotechnology Regulatory Services. Accessed electronically 20 Feb 2004, http:// www.aphis.usda.gov/brs/conformiance9.html

United States Environmental Protection Agency [USEPA] (1992) Guidelines for Exposure Assessment. FRL-4129-5.
Exposure Assessment Group, Office of Health and Environmental Assessment, USEPA, Washington, DC

United States Environmental Protection Agency [USEPA] (2002) EPA Settles with Two Biotech Seed Production Companies. USEPA Region 9 Press Release December 13, 2002

United States Environmental Protection Agency [USEPA] (2003) AERMOD: Latest features and evaluation results. EPA-454/R-03-003 June 2003

University of Arkansas Cooperative Extension Service [UACES] (2003) Corn harvesting. Accessed electronically 9 May 2004, http://www.aragriculture.org/agengineering/ harvesting/corn/default.asp

Vose D (2000) Risk analysis: A Quantitative Guide. 2nd edn, John Wiley, New York

Wolt JD, Peterson RKD (2000) Agricultural biotechnology and societal decision-making: The role of risk analysis. AgBioForum 3: 291-298

Wolt JD, Peterson RKD, Bystrak P, Meade T (2003) A screening level approach for nontarget insect risk assessment: Transgenic Bt maize pollen and the monarch butterfly (Lepidoptera: Danaidae). Environ. Entomol. 32: 237-246 